\section{Wie gefährlich ist der Schnitt in das Melanom?}

Die Exzisionsbiopsie bleibt die Methode der Wahl bei verdächtigen

Pigmentläsionen. Ist keine komplette Entfernung möglich, dann stellt die

Shave-Biopsie (Saucerization) eine akzeptable Alternative dar - aktuellen

Studiendaten zufolge wird das Überleben der Melanompatienten dadurch nicht beeinflusst.

U S-amerikanische Kollegen haben die Daten aller Patienten ausgewertet, die in den Jahren 2000-2008 wegen eines Melanoms am Baylor College of Medicine in Houston behandelt worden waren. Bei $14,2 \%$ der 479 Fälle war eine Exzisionsbiopsie (mittlere Breslow-Tiefe 2,28 mm) erfolgt, bei $25,7 \%$ der Patienten eine Stanzbiopsie (mittlere Breslow-Tiefe 0,92 $\mathrm{mm}$ ) und bei $60,1 \%$ eine "Saucerization“ durch tiefe Aushöhlung mit dem Skalpell (Shave- und Deep-Shave-Biopsien), die mittlere Breslow-Tiefe lag hier bei 0,74 mm.

Der Tumor wurde unterschiedlich häufig verletzt: Bei der Exzisionsbiopsie betrug die Transsektionsrate $1,5 \%$, bei der Stanzbiopsie 4,1\%. Die Verletzung des
Tumors durch Saucerization war in 9,0\% dokumentiert. Da sich bei einigen Patienten der Melanomverdacht nicht bestätigte, lag die tatsächliche Rate nach Saucerization nur bei $5,5 \%$.

Insgesamt zeigten sich keine signifikanten Unterschiede der Mortalitätsraten zwischen Patienten, deren Melanom bei der Diagnostik angeschnitten wurde und den Patienten ohne Transsektion (krankheitsfreies Überleben: 833 vs. 911 Tage, Gesamtüberleben; 1.012 vs. 1.074 Tage).

Fazit: Die Art der Biopsie hatte keinen Einfluss auf das Überleben der Patienten. Da Biopsien durch Saucerization häufig die gesamte Läsion erfassen, sind sie bei

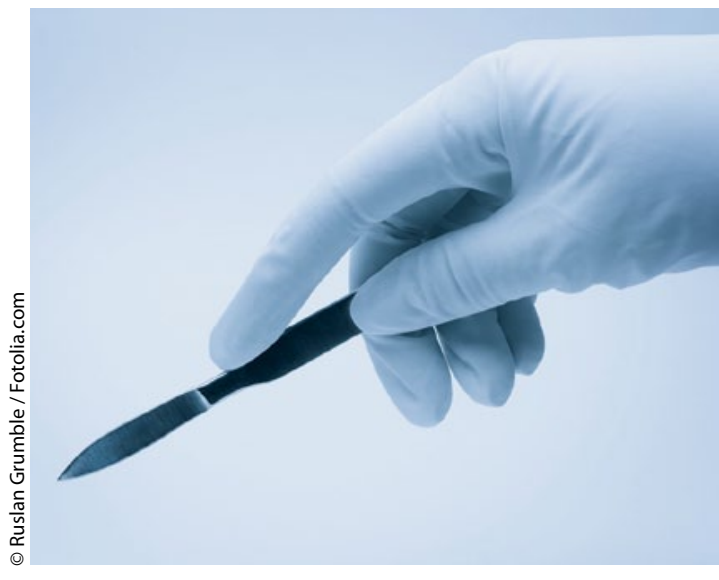

flacheren, kleinen Veränderungen eine akzeptable Alternativen zur tiefen chirurgischen Biopsie, so die Autoren.

Dr. Christine Starostzik

Mir $\mathrm{M}$ et al. The rate of melanoma transsection with various biopsy techniques and the influence of tumor transection on patient survival. J Am Acad Dermatol 2012, Sep 8 [Epub ahead of print]

\title{
Psoriasis: Daten sprechen für Apremilast als effektive Therapie
}

\begin{abstract}
Apremilast zählt zu den „Small Molcules" und ist ein Inhibitor des Enzyms Phosphodiesterase 4 (PDE-4). In einer Phase-llb-Studie hat sich die Substanz als gut wirksam gegen mäßige und schwere Formen der Psoriasis erwiesen.
\end{abstract}

$\mathrm{D}$ as intrazelluläre Enzym PDE-4 baut zyklisches Adenosin-Monoposphat (cAMP) ab und erhöht die Produktion proentzündlicher Zytokine. Indikationsgebiete für PDE-4-hemmende Substanzen sind neben der Psoriasis auch chronischobstruktive Lungenerkrankungen und Arthritiden.

An der multizentrischen Dosisfindungsstudie beteiligten sich 352 Patienten von 35 US-amerikanischen und kanadischen Einrichtungen. Die Teilnehmer litten seit mindestens einem halben Jahr an mäßiger bis schwerer Psoriasis (Psoriasis Area and Severity Index, PASI $\geq 12$, mindestens $10 \%$ der Hautfläche betroffen). Randomisiert wurden sie vier Gruppen zugewiesen, in denen sie Apremilast in drei unterschiedlichen Dosierungen
(10, 20, $30 \mathrm{mg}$ ) oder Placebo zweimal täglich einzunehmen hatten. Primärer Endpunkt der Untersuchung war der Anteil der Patienten, die nach 16 Wochen eine Reduktion des PASI um mindestens $75 \%$ erreichten.

Nach vier Monaten zeigten 6\% der Probanden in der Placebogruppe ein PASI-75-Ansprechen. Für den verschiedenen Verumgruppen-Dosierungen lagen die Patientenanteile mit einem PASI75 -Ergebnis bei $11 \%$ (10 mg), bei $29 \%$ $(20 \mathrm{mg})$ und bei $41 \%(30 \mathrm{mg})$. Ein signifikanter Unterschied im Vergleich zu Placebo ergab sich für die 20- und die 30-mg-Dosis $(\mathrm{p}<0,0001)$. Der Effekt von $10 \mathrm{mg}$ Apremilast unterschied sich nicht statistisch bedeutsam vom Ergebnis der Placebobehandlung.
96\% der dokumentierten unerwünschten Wirkungen waren leichter bis mäßiger Ausprägung, wobei mit einer Übelkeit, Infekte der oberen Atemwege, Diarrhö, Nasopharyngitis, Kopfschmerzen, Arthralgie, Gastroenteritis und Dyspepsie mit einer Häufigkeit von mindestens 5\% auftraten. Keiner der schweren Zwischenfälle, darunter Malignome und kardiovaskuläre Ereignisse, wurde von den Forschern auf die Wirksubstanz zurückgeführt. Opportunistische Infektionen waren nicht festzustellen.

Fazit: Der PDE-4-Hemmer Apremilast ist nach diesen Ergebnissen sicher und effektiv bei mäßigen bis schweren Formen der Psoriasis. In der vorliegenden Dosisfindungsstudie zeigten sich Dosierungen von 20 und $30 \mathrm{mg}$ zweimal täglich dem Placebopräparat signifikant und deutlich überlegen.

Dr. Robert Bublak

Papp K et al. Efficacy of apremilast in the treatment of moderate to severe psoriasis: a randomised controlled trial. Lancet 2012; 380: 738-46 